\title{
Pretreatment elevated prognostic nutritional index predicts a favorable prognosis in patients with prostate cancer
}

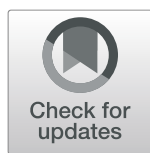

Bin $\mathrm{Li}^{1}$, Zheng $\mathrm{Lu}^{1}$, Shengzheng Wang ${ }^{2}$, Junqing $\mathrm{Hou}^{3}$, Gang Xia ${ }^{1}$, Heng Li ${ }^{1}$, Bo Yin ${ }^{1}$ and Wei $\mathrm{Lu}^{1,3^{*}}$ (D)

\begin{abstract}
Background: The prognostic nutritional index (PNI), an immunity and nutrition based prognostic score, was correlated with clinical outcomes in different tumors. However, the prognostic significance of PNI has not been investigated in hormone sensitive prostate cancer (PCa). The objective of this study was to determine the prognostic significance of PNI in hormone sensitive PCa.
\end{abstract}

Methods: Two hundred eighty PCa patients undergoing androgen deprivation therapy (ADT) as first line therapy at three centers were enrolled. The serum albumin levels and peripheral lymphocyte count were measured at the time of diagnosis. PNI was calculated as $10 *$ serum albumin $(\mathrm{g} / \mathrm{dL})+0.005 *$ total lymphocyte count (per mm3). Patients were categorized in two groups using a cut-off point of 50.2 as calculated by the receiver-operating curve analysis. Univariate and multivariate cox regression analyses were performed to evaluate PNI as a favorable prognostic factor for progression-free survival (PFS), cancer-specific survival (CSS) and overall survival (OS). Prognostic accuracy was evaluated with the Harrell concordance index.

Results: Multivariate analyses identified PNI as an independent prognostic indicator with respect to PFS (hazard ratio $(H R)=0.521, p=0.001)$, CSS ( $H R=0.421, p=0.002)$ and OS ( $H R=0.429, p=0.001)$. Patients with elevated PNI had better clinical outcomes. The addition of PNI to the final models improved predictive accuracy (c-index: 0.758 , 0.830 and 0.782 ) for PFS, CSS and OS compared with the clinicopathological base models (c-index: 0.736, 0.801 and 0.752), which included Gleason score and incidence of metastasis.

Conclusions: Elevated pretreatment PNI was a favorable prognostic indicator for PCa patients treated with ADT.

Keywords: Prostate cancer, Prognostic nutritional index, Prognostic factor, Survival

\section{Background}

Prostate cancer $(\mathrm{PCa})$ is the most common malignance in men from United States [1]. Androgen deprivation therapy (ADT) is one of the initial treatments for patients with $\mathrm{PCa}[2]$.

In the present, the prognostic evaluation of PCa relies on the common indicators, such as PSA levels, TNM

\footnotetext{
* Correspondence: luweimed@163.com

'Department of Urology, Xinyang Central Hospital, 1 Siyi Road, Shihe District, Xinyang, Henan 464000, People's Republic of China

${ }^{3}$ Clinical Medical College of Henan University, Kaifeng, Henan, China

Full list of author information is available at the end of the article
}

stage. Recent studies indicate that in addition to tumor cellular differentiation and biological behavior, the prognosis of cancer is also related to the immunological and nutritional status [3-6]. Jang WS et al. [7] reported that preoperative neutrophil to lymphocyte ratio was an independent prognostic marker for patients with PCa. Similarly, Langsenlehner $\mathrm{T}$ et al. [8] showed a significant association between platelet to lymphocyte ratio and prognosis of $\mathrm{PCa}$ patients who underwent radiation therapy. Serum albumin level, which is commonly used to assess the nutritional status, is an important prognostic

(c) The Author(s). 2020 Open Access This article is licensed under a Creative Commons Attribution 4.0 International License, which permits use, sharing, adaptation, distribution and reproduction in any medium or format, as long as you give appropriate credit to the original author(s) and the source, provide a link to the Creative Commons licence, and indicate if changes were made. The images or other third party material in this article are included in the article's Creative Commons licence, unless indicated otherwise in a credit line to the material. If material is not included in the article's Creative Commons licence and your intended use is not permitted by statutory regulation or exceeds the permitted use, you will need to obtain permission directly from the copyright holder. To view a copy of this licence, visit http://creativecommons.org/licenses/by/4.0/ The Creative Commons Public Domain Dedication waiver (http://creativecommons.org/publicdomain/zero/1.0/) applies to the data made available in this article, unless otherwise stated in a credit line to the data. 
factor in advanced cancer [9]. The specific relationship between nutrition and PCa still remained unclear [10], however, Kenfield SA et al. [11] reported that a healthy lifestyle might lower risk of lethal $\mathrm{PCa}$, and Sejima $\mathrm{T}$ et al. [12] showed that low preoperative serum albumin was associated with lymph node metastases and biochemical recurrence of $\mathrm{PCa}$ in patients with radical prostatectomy, which indicated an important role of nutritional status in the prognosis of $\mathrm{PCa}$.

The prognostic nutritional index (PNI), which is calculated on the basis of serum albumin levels and peripheral lymphocyte count, is a simple and comprehensive index to reflect the immunological and nutritional status. PNI was originally described by Onodera et al. [13] to evaluate the perioperative immunonutritional status and risk of post-surgical complications, and has been validated as an independent powerful prognostic factor for various types of cancer, including renal cell cancer [14], breast cancer [15], gastric cancer [16], colorectal cancer [17], hepatocellular carcinoma [18], pancreatic cancer [19], small cell and non-small cell lung cancer [20, 21], esophageal squamous cell cancer [22], and castration-resistant prostate cancer [23]. However, the value of PNI in the prognosis of hormone sensitive $\mathrm{PCa}$ has not been assessed.

Serum albumin levels and peripheral lymphocyte count are routinely performed before treatment for patients with PCa, and PNI is easy to be calculated. Accordingly, we investigated the prognostic significance of PNI in a cohort of hormone sensitive PCa patients from three Chinese centers.

\section{Methods}

\section{Patient population}

Three hundred twenty-two prostate cancer ( $\mathrm{PCa}$ ) patients underwent continuous ADT as first line therapy between Jan 2013 and Dec 2016 at three centers, including castration and antiandrogen therapy. Study exclusion criteria were inflammatory diseases, hepatopathy, autoimmune diseases, hematologic diseases, other types of cancer, cardiovascular and cerebrovascular diseases, and those patients lost to follow-up. Data from 280 patients were ultimately analyzed in this study. The study protocol was approved by the Ethics Committees from Xinyang Central Hospital, The First Affiliated Hospital of Zhengzhou University, and Clinical Medical College of Henan University, and all patients provided written informed consents.

Clinical and pathological characteristics were collected. All blood samples were measured before the prostate biopsy. PNI was calculated as 10 * serum albumin $(\mathrm{g} / \mathrm{dL})+$ $0.005 *$ total lymphocyte count (per mm3) [13]. The clinical metastasis was evaluated with radionuclide bone scan. PCa patients were divided into low-, intermediate-, and high-risk groups according to the EAU-ESTROSIOG guidelines [24].

Follow-up was assessed from the day of treatment to the day of death or last follow-up visit (Jun 2019). Overall survival (OS) was calculated from the day of treatment to the day of death or the last follow-up visit. Cancer specific survival (CSS) was defined as time between the day of treatment and date of death for PCa or the last follow-up visit. Progression was considered as castration resistance or death, and the castration resistance was evaluated according to the EAU-ESTRO-SIOG guideline [25].

\section{Statistical analysis}

SPSS, version 19.0 was used to analyze the data. The receiver operating characteristic (ROC) curve analysis was performed to select the most appropriate cut-off point for PNI to separate the patients at high risk of cancerrelated death. The correlation between PNI and clinical characteristics was assessed by the Wilcoxon signed rank tests for continuous variables, and the chi-squared tests for categorical variables. The Kaplan-Meier method was used for PFS, CSS and OS estimation, the log-rank test was taken to investigate the difference on survival. Stratified analyses were performed on the stratification of Gleason score or incidence of metastasis. The univariate and multivariate stepwise cox proportional hazard regression model was used to evaluate the effect of PNI on survival. The c-index was calculated to evaluate the predictive accuracy of model on basic clinical variables and for comparison after the addition of PNI using the R package "survival" [26]. A two tailed value of $P<0.05$ was considered statistically significant.

\section{Results}

Two hundred eighty patients were ultimately included in this study. Table 1 presented the baseline clinical characteristics of the patients. The median age of the patients was 76 years old (IQR, 67.25-79), and 131(46.79\%) patients had bone metastasis at presentation. After a median follow-up of 46.0 months, 129 (46.07\%) patients experienced disease progression, and 78 (27.86\%) patients died, including 63 (22.50\%) patients died of PCa.

The best cut-off value of PNI for CSS as determined by ROC curve was 50.2. The patients in the low PNI group were significantly older than that in the elevated PNI group $(p<0.001)$. However, serum PSA level, Gleason score, risk stratification and incidence of metastasis were comparable in the two groups $(p>0.05)$. (Table 2).

The Kaplan-Meier analysis showed significantly better PFS, CSS and OS in patients with elevated PNI than patients with decreased PNI (each $P<0.01$, Fig. 1). As shown in Fig. 2 and Fig. 3, in the group of Gleason score $>7$ or bone metastasis, the patients with elevated 
Table 1 Clinical characteristics of prostate cancer patients treated with ADT $(n=280)$

\begin{tabular}{|c|c|}
\hline Parameters & No. of patients(\%) \\
\hline Age (median, interquartile range), years & $76(67.25-79)$ \\
\hline PSA (median, interquartile range), $\mu \mathrm{g} / \mathrm{L}$ & $91.20(29.26-189.00)$ \\
\hline \multicolumn{2}{|l|}{ Gleason Score } \\
\hline$<7$ & $28(10.00)$ \\
\hline $3+4$ & $42(15.00)$ \\
\hline $4+3$ & $68(24.29)$ \\
\hline 8 & $89(31.78)$ \\
\hline 9 & $50(17.86)$ \\
\hline 10 & $3(1.07)$ \\
\hline \multicolumn{2}{|l|}{ Metastasis } \\
\hline No & $149(53.21)$ \\
\hline Yes & $131(46.79)$ \\
\hline \multicolumn{2}{|l|}{ Risk Stratification } \\
\hline Low & $1(0.36)$ \\
\hline Intermediate & $26(9.28)$ \\
\hline High & $253(90.36)$ \\
\hline PNI (median, interquartile range) & $50.05(46.46-53.55)$ \\
\hline Progression-free survival & $129(46.07)$ \\
\hline Cancer-specific survival & $63(22.50)$ \\
\hline Overall survival & $78(27.86)$ \\
\hline \multicolumn{2}{|l|}{ Types of ADT } \\
\hline Orchiectomy + anti-androgen therapy & $272(97.14)$ \\
\hline LHRN-a + anti-androgen therapy & $8(2.86)$ \\
\hline $\begin{array}{l}\text { Follow-up time (median, interquartile range), } \\
\text { months }\end{array}$ & $46.00(31.00-59.00)$ \\
\hline
\end{tabular}

Abbreviations: PSA Prostate-specific antigen; PNI Prognostic nutritional index; $A D T$ Androgen deprivation therapy; LHRN-a Luteinizing hormone releasing hormone-a

PNI had better PFS, CSS and OS (each $P<0.05$ ). However, in the group of Gleason score $\leq 7$ or nonmetastasis, the prognosis was comparable in the two groups (each $P>0.05$ ).

Univariate analyses demonstrated that elevated Gleason score, high incidence of metastasis, decreased PNI were associated with a worse PFS, CSS and OS (each $P<0.01$ ), decreased age was a favorable predictor for PFS, not for
CSS and OS (Table 3). Multivariate analysis indicated that age, Gleason score, incidence of metastasis and PNI were independent prognostic factors for PFS, while Gleason score, incidence of metastasis and PNI were independent prognostic factors for CSS and OS. The HRs of PNI were 0.521 (95\% CI 0.355-0.765) for PFS, 0.421 (95\% CI $0.243-$ 0.728 ) for CSS and 0.429 (95\% CI $0.263-0.698$ ) for OS, respectively (Table 4).

The c-index of the base model, including Gleason score and incidence of metastasis, for PFS, CSS and OS was $73.6 \%$ (IQR, 70.3-76.4\%), 80.1\% (IQR, 76.0-82.3\%) and $75.2 \%$ (IQR, 71.4-78.1\%). After the addition of PNI, the c-index for PFS, CSS and OS was 75.8\% (IQR, 72.678.1\%), 83.0\% (IQR, 79.7-85.4\%) and 78.2\% (IQR, 74.4$81.7 \%)$, respectively which was higher than that of the base model (each $p<0.001$ ).

\section{Discussion}

The incidence of prostate cancer (PCa) in China is increasing in recent years [27], and the proportion of aged or advanced or metastatic $\mathrm{PCa}$ is higher than that in western countries, androgen deprivation therapy is the main treatment for these patients. Almost all the patients would experience castration resistance after ADT, to search for risk factors that could predict the prognosis of PCa patients receiving ADT is of great importance.

The serum albumin levels and peripheral lymphocyte count are routinely measured before the treatment of $\mathrm{PCa}$. In this large cohort of PCa patients receiving ADT from three centers, we discovered that pretreatment elevated prognostic nutritional index (PNI) was a favorable prognostic indicator, independent of clinicopathological features and could significantly improve the predictive accuracy for prognosis, indicating it should be considered as an additional biomarker for PCa patients' individual prognostic risk assessment.

The mechanism responsible for this observation remains unclear. The PNI represents a marker of immunity and nutrition. A decreased PNI reflects both malnutrition status and weak lymphocyte mediated antitumor immune response, which may both contribute to cancer progression and poor outcome. Malnutrition is highly prevalent in cancer patients, and may produce a great deal of negative

Table 2 Clinical characteristics of prostate cancer patients according to PNI

\begin{tabular}{llll}
\hline Parameters & \multicolumn{1}{l}{ PNI } & \multirow{2}{*}{$P$-value } \\
\cline { 2 - 3 } & $<50.2(n=14652.14 \%) \geq 50.2(n=134$ 47.86\%) & $75(64-78)$ & $<0.001$ \\
Age (median, interquartile range), years & $77(72-81)$ & $76.04(26.60-135.61)$ & 0.171 \\
PSA (median, interquartile range), $\mu \mathrm{g} / \mathrm{L}$ & $100.00(35.5-216.00)$ & $72 / 62$ & 0.154 \\
Gleason Score( $\leq 7 />7)$ & $66 / 80$ & $77 / 57$ & 0.172 \\
Metastasis (no/yes) & $72 / 74$ & $14 / 120$ & 0.662 \\
Risk Stratification (lowintermediate/high) & $13 / 133$ & & \\
\hline
\end{tabular}

Abbreviations: PSA Prostate-specific antigen; PNI Prognostic nutritional index 

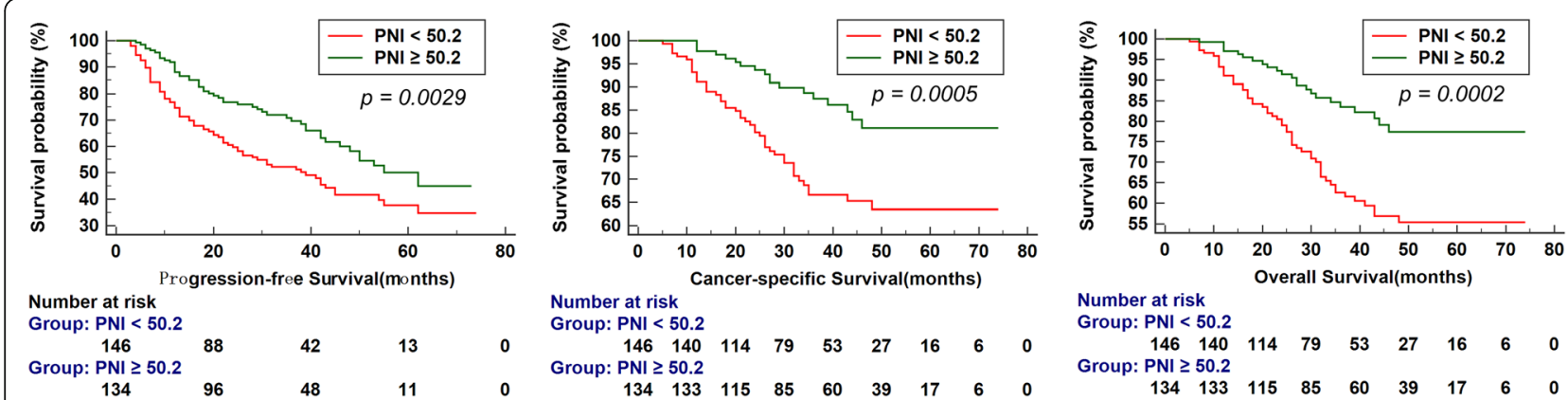

Fig. 1 Kaplan-Meier curves for survival of prostate cancer patients according to PNI. a. Progression-free survival (PFS), b. Cancer-specific survival (CSS) and c. Overall survival (OS)

consequences, such as impaired immune functions and quality of life, a higher degree of treatment-related toxicity and complications, reduced response to cancer treatment, lower activity level and shortened overall survival [28]. Sakurai $\mathrm{K}$ et al. [16] reported an association between low PNI with unfavorable outcomes for patients with gastric cancer at stages 1 and 2, and they speculated that malnutrition and immune compromise might promote proliferation and metastasis of peripheral blood circulating tumor cells (CTCs) in patients with early gastric cancer, increasing the risk of tumor recurrence. Therefore, malnutrition should be appropriately managed by structured collaboration between oncologists and clinical nutrition specialists [29]. Meta-analysis revealed that higher serum albumin level was associated with a better survival in patients with different tumors [30]. As part of systemic inflammatory response to the tumor or from the tumor itself, proinflammatory cytokines including interleukin-1, IL-6, and necrosis factor a are released, which may modulate albumin synthesis by hepatocytes [31, 32], these cytokines are crucial for malignant transformation, neoangiogenesis and cancer progression [31], thus nutritional status could serve as a good indicator of prognosis for cancer. As to lymphocyte, it is one of the components of the immune system, which could inhibit tumor progression [33]. The HPV+ oropharyngeal cancer patients with pretreatment high circulating lymphocytes were reported with better prognosis [34]. Roth MD et al. [35] established a humanized prostate cancer model for
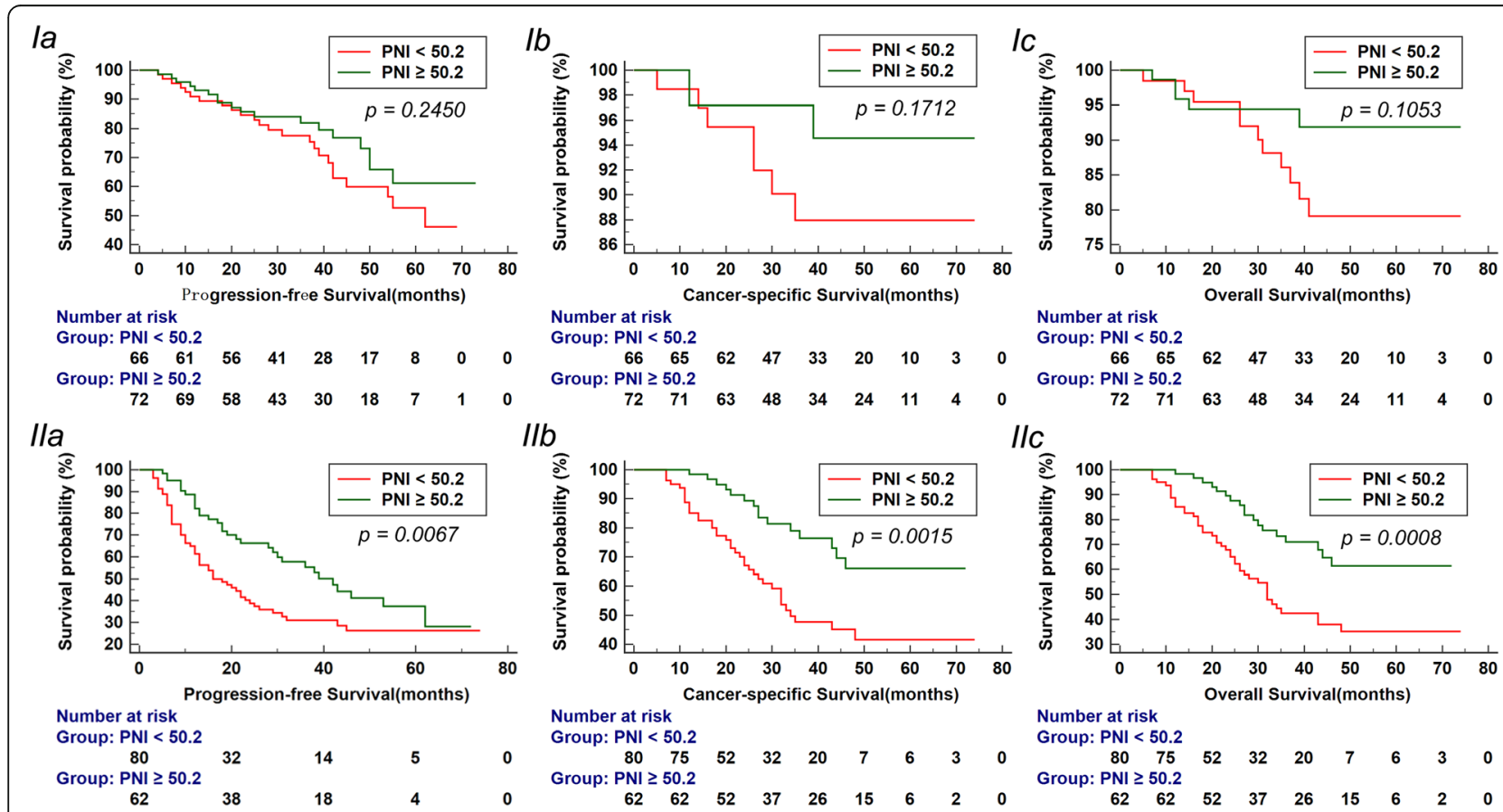

IIC

Fig. 2 Kaplan-Meier survival curves stratified by PNI in prostate cancer patients with Gleason score $\leq 7(I)$ and Gleason score $>7(I I)$. a. Progressionfree survival (PFS), b. Cancer-specific survival (CSS) and c. Overall survival (OS) 

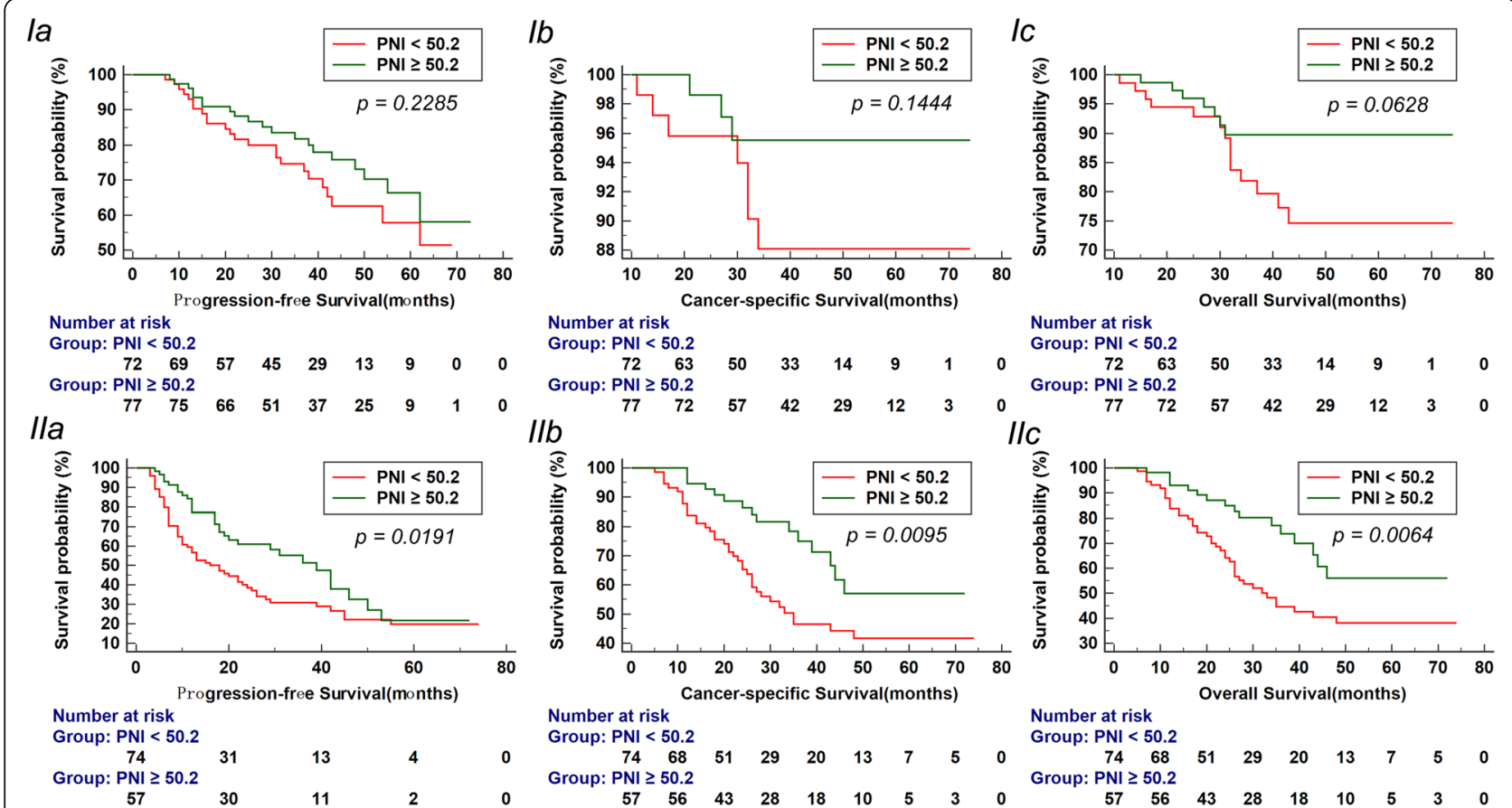

Fig. 3 Kaplan-Meier survival curves stratified by PNI in prostate cancer patients with non-metastasis(I) and metastasis (II). a. Progression-free survival (PFS), b. Cancer-specific survival (CSS) and c. Overall survival (OS)

understanding and manipulating tumor infiltrating lymphocytes, and found that the presence of tumor infiltrating lymphocyte was associated with a marked slowing of tumor growth although did not result in tumor eradication.

To date, it is controversial about the prognostic value of PNI in the clinical studies, some studies say yes [1423], and others say no [36-38]. Sun K et al. [39] made a meta-analysis and showed that elevated PNI was a favorable predictor for OS, and the presence of post-operative complications, which indicated the association of decreased PNI with adverse clinical outcomes. The prognostic impact of PNI in many other cancers has been reported, on the contrary, its value in $\mathrm{PCa}$ is poorly investigated.

Table 3 Univariate analyses of various clinical parameters in prostate cancer patients

\begin{tabular}{|c|c|c|c|c|c|c|}
\hline \multirow[t]{2}{*}{ Parameters } & \multicolumn{2}{|c|}{ Progression-Free Survival } & \multirow{2}{*}{$\begin{array}{l}\text { Cancer-Specific Survival } \\
\text { HR }(95 \% \mathrm{Cl}) P \text {-value }\end{array}$} & & \multicolumn{2}{|l|}{ Overall Survival } \\
\hline & $\mathrm{HR}(95 \% \mathrm{Cl})$ & $P$-value & & & $\mathrm{HR}(95 \% \mathrm{Cl})$ & $P$-value \\
\hline Age (years) & $0.969(0.948-0.990)$ & 0.004 & $0.971(0.944-1.000)$ & 0.050 & $0.988(0.962-1.015)$ & 0.396 \\
\hline PSA $(\mu \mathrm{g} / \mathrm{L})$ & $1.000(1.000-1.001)$ & 0.228 & $1.000(1.000-1.001)$ & 0.273 & $1.000(0.999-1.001)$ & 0.611 \\
\hline Gleason Score & & $<0.001$ & & $<0.001$ & & $<0.001$ \\
\hline$\leq 7$ & 1 & & 1 & & 1 & \\
\hline$>7$ & $2.907(2.006-4.211)$ & & $6.425(3.265-12.643)$ & & $4.740(2.733-8.220)$ & \\
\hline Metastasis & & $<0.001$ & & $<0.001$ & & $<0.001$ \\
\hline No & 1 & & 1 & & 1 & \\
\hline Yes & $3.528(2.443-5.096)$ & & $7.920(4.024-15.589)$ & & $4.122(2.496-6.809)$ & \\
\hline Risk Stratification & & 0.023 & & 0.069 & & 0.095 \\
\hline Lowintermediate & 1 & & 1 & & 1 & \\
\hline High & $2.428(1.133-5.205)$ & & $24.120(0.779-746.903)$ & & $2.357(0.861-6.447)$ & \\
\hline PNI & & 0.004 & & 0.001 & & $<0.001$ \\
\hline$<50.2$ & 1 & & 1 & & 1 & \\
\hline$\geq 50.2$ & $0.590(0.414-0.841)$ & & $0.391(0.226-0.676)$ & & $0.407(0.250-0.662)$ & \\
\hline
\end{tabular}


Table 4 Multivariate analyses of various clinical parameters in prostate cancer patients

\begin{tabular}{|c|c|c|c|c|c|c|}
\hline \multirow[t]{2}{*}{ Parameters } & \multicolumn{2}{|c|}{ Progression-Free Survival } & \multicolumn{2}{|c|}{ Cancer-Specific Survival } & \multicolumn{2}{|l|}{ Overall Survival } \\
\hline & HR (95\% Cl) & $P$-value & HR $(95 \% \mathrm{Cl})$ & $P$-value & HR (95\% Cl) & $P$-value \\
\hline Age (years) & $0.975(0.953-0.997)$ & 0.028 & & - & & - \\
\hline Gleason Score & & $<0.001$ & & $<0.001$ & & $<0.001$ \\
\hline$\leq 7$ & 1 & & 1 & & 1 & \\
\hline$>7$ & $2.392(1.638-3.495$ & & $5.073(2.569-10.020)$ & & 4.003 (2.298-6.974) & \\
\hline Metastasis & & $<0.001$ & & $<0.001$ & & $<0.001$ \\
\hline No & 1 & & 1 & & 1 & \\
\hline Yes & $2.930(2.021-4.247)$ & & 6.091 (3.086-12.024) & & 3.245 (1.957-5.378) & \\
\hline PNI & & 0.001 & & 0.002 & & 0.001 \\
\hline$<50.2$ & 1 & & 1 & & 1 & \\
\hline$\geq 50.2$ & $0.521(0.355-0.765)$ & & $0.421(0.243-0.728)$ & & $0.429(0.263-0.698)$ & \\
\hline
\end{tabular}

Abbreviations: HR Hazard ratio; Cl Confidence interval; PSA Prostate-specific antigen; PNI Prognostic nutritional index

As far as I know, this is the first study to address the prognostic value of PNI in hormone sensitive $\mathrm{PCa}$, and it revealed that elevated PNI was a favorable predictor for clinical outcomes. Moreover, we performed a stratified analysis and built a prognostic predictive model. In stratified analysis, we showed an association between decreased PNI and poor outcomes in patients with Gleason score $>7$ or bone metastasis. In the patients with Gleason score $\leq 7$ or non-metastasis, PNI could not predict the prognosis due to the small percentage of patients who reached the endpoints in this subgroup. The predictive accuracy for PFS, CSS and OS was significantly higher after the addition of PNI. In the meta-analysis by Sun K et al. [39], PNI was correlated with invasion depth and lymph node metastasis in gastric cancer, and TNM stage was the only clinicopathological feature associated with PNI in colorectal carcinoma. From the results of meta-analysis and aforementioned explanation, it seems that PNI should be associated with the clinicopathological features, however, the tumor features including serum PSA level, Gleason score, risk stratification and incidence of metastasis were similar in the two groups in our study, but similar to the report by Watanabe $\mathrm{M}$ et al. [40], the patients with low PNI had elevated age.

The same as all the retrospective studies, the first limitation of our study is its design. In an attempt to reduce the factors of influencing albumin levels and lymphocyte counts, we set up strict enrollment criteria, however, it is impossible to completely rule out other conditions that might cause immunological and nutritional changes in patients with $\mathrm{PCa}$. Second, $\mathrm{T}$ and $\mathrm{N}$ stage were not showed for that $15 \% \mathrm{PCa}$ patients with serum PSA level $>20 \mu \mathrm{g} / \mathrm{L}$ refused CT or MRI scan to evaluate tumor invasion depth and determine whether there was lymph node metastasis. Nonetheless, our data clearly indicated that PNI was an independent prognostic indicator for PFS, CSS and OS in PCa patients.

\section{Conclusions}

Pretreatment PNI might be a novel prognostic predictor for PCa patients treated with ADT, the lower the PNI, the worse the prognosis. This biomarker should be considered in future prognostic risk assessment to improve the predictive accuracy.

\section{Supplementary information}

Supplementary information accompanies this paper at https://doi.org/10. 1186/s12885-020-06879-1.

\section{Additional file 1.}

\section{Acknowledgements}

We sincerely thank the patients for their participation in this study.

\section{Authors' contributions}

LB and LW were responsible for the design of the study. LB, LW and LZ contributed to the manuscript composition, submission and revision. WSZ, $H J Q, X G, L H$ and YB participated in the data collection, interpretation and discussion. LB and LZ participated in the data analysis and manuscript mapping. All authors have contributed to and approved the final manuscript.

\section{Funding}

None.

Availability of data and materials

You can see the data and materials in additional file named "original data.xIsx".

Ethics approval and consent to participate

The study protocol was approved by the Ethics Committees from Xinyang Central Hospital, The First Affiliated Hospital of Zhengzhou University, and Clinical Medical College of Henan University. Informed consents to participate in this study have been obtained from all participants.

Consent for publication

We had obtained the consents to publish from the participants to report individual patients' data in any form (including images, videos, voice recordings etc.)

Competing interests

The authors declare no competing interests. 


\section{Author details}

'Department of Urology, Xinyang Central Hospital, 1 Siyi Road, Shihe District, Xinyang, Henan 464000, People's Republic of China. ${ }^{2}$ Department of Urology, The First Affiliated Hospital of Zhengzhou University, Zhengzhou, Henan, China. ${ }^{3}$ Clinical Medical College of Henan University, Kaifeng, Henan, China.

Received: 9 December 2019 Accepted: 19 April 2020

Published online: 29 April 2020

\section{References}

1. Siegel RL, Miller KD, Jemal A. Cancer statistics, 2019. CA Cancer J Clin. 2019; 69(1):7-34.

2. Wein AJ, Kavaussi LR, Novick AC, Partin AW, Peters CA, Nelson JB, et al. Campbell-wash Urology,10th edition. Philadelphia: Elsevier; 2012. p. 2934-53.

3. Hutcheson J, Balaji U, Porembka MR, Wachsmann MB, McCue PA, Knudsen ES, et al. Immunological and metabolic features of pancreatic ductal adenocarcinoma define prognostic subtypes of disease. Clin Cancer Res. 2016;22(14):3606-17.

4. Santoni M, Buti S, Conti A, Porta C, Procopio G, Sternberg CN, et al. Prognostic significance of host immune status in patients with late relapsing renal cell carcinoma treated with targeted therapy. Target Oncol. 2015:10(4):517-22.

5. Sánchez-Lara K, Turcott JG, Juárez E, Guevara P, Núñez-Valencia C, OñateOcaña LF, et al. Association of nutrition parameters including bioelectrical impedance and systemic inflammatory response with quality of life and prognosis in patients with advanced non-small-cell lung cancer: a prospective study. Nutr Cancer. 2012;64(4):526-34.

6. Gu W, Zhang G, Sun L, Ma Q, Cheng Y, Zhang H, et al. Nutritional screening is strongly associated with overall survival in patients treated with targeted agents for metastatic renal cell carcinoma. J Cachexia Sarcopenia Muscle. 2015;6(3):222-30.

7. Jang WS, Cho KS, Kim KH, Yoon CY, Kang YJ, Lee JY, et al. Prognostic impact of preoperative neutrophil-to-lymphocyte ratio after radical prostatectomy in localized prostate cancer. Prostate Cancer Prostatic Dis. 2016;19(3):298-304.

8. Langsenlehner T, Pichler M, Thurner EM, Krenn-Pilko S, Stojakovic T, Gerger A, et al. Evaluation of the platelet-to-lymphocyte ratio as a prognostic indicator in a European cohort of patients with prostate cancer treated with radiotherapy. Urol Oncol. 2015;33(5):201.e9-16.

9. Fiala O, Pesek M, Finek J, Racek J, Minarik M, Benesova L, et al. Serum albumin is a strong predictor of survival in patients with advanced-stage non-small cell lung cancer treated with erlotinib. Neoplasma. 2016;63(3): 471-6.

10. Masko EM, Allott EH, Freedland SJ. The relationship between nutrition and prostate cancer: is more always better? Eur Urol. 2013;63(5):810-20.

11. Kenfield SA, Batista JL, Jahn JL, Downer MK, Van Blarigan EL, Sesso HD, et al. Development and application of a lifestyle score for prevention of lethal prostate cancer. J Natl Cancer Inst. 2015; 108(3). pii: djv329.

12. Sejima $T$, Iwamoto $H$, Masago $T$, Morizane $S$, Yao A, Isoyama $T$, et al. Low pre-operative levels of serum albumin predict lymph node metastases and ultimately correlate with a biochemical recurrence of prostate cancer in radical prostatectomy patients. Cent European J Urol. 2013;66(2):126-32.

13. Onodera T, Goseki N, Kosaki G. Prognostic nutritional index in gastrointestinal surgery of malnourished cancer patients. Nihon Geka Gakkaishi Zasshi. 1984;85(9):1001-5.

14. Hofbauer SL, Pantuck AJ, de Martino M, Lucca I, Haitel A, Shariat SF, et al. The preoperative prognostic nutritional index is an independent predictor of survival in patients with renal cell carcinoma. Urol Oncol. 2015;33(2):68.e1-7.

15. Yang Z, Zhang B, Hou L, Xie Y, Cao X. Pre-operative prognostic nutritional index predicts the outcomes for triple-negative breast cancer. Tumour Biol. 2014;35(12):12165-71.

16. Sakurai K, Ohira M, Tamura T, Toyokawa T, Amano R, Kubo N, et al. Predictive potential of preoperative nutritional status in long-term outcome projections for patients with gastric Cancer. Ann Surg Oncol. 2016;23(2):525-33.

17. Jian-Hui C, Iskandar EA, Cai SI, Chen CQ, Wu H, Xu JB, et al. Significance of Onodera's prognostic nutritional index in patients with colorectal cancer: a large cohort study in a single Chinese institution. Tumour Biol. 2016;37(3): 3277-83.

18. Chan AW, Chan SL, Wong GL, Wong WW, Chong CC, Lai PB, et al. Prognostic nutritional index (PNI) predicts tumor recurrence of very early/early stage hepatocellular carcinoma after surgical resection. Ann Surg Oncol. 2015. 22(13):4138-48.
19. Watanabe J, Otani S, Sakamoto T, Arai Y, Hanaki T, Amisaki M, et al. Prognostic indicators based on inflammatory and nutritional factors after pancreaticoduodenectomy for pancreatic cancer. Surg Today. 2016;46(11):1258-67.

20. Hong S, Zhou T, Fang W, Xue C, Hu Z, Qin T, et al. The prognostic nutritional index (PNI) predicts overall survival of small-cell lung cancer patients. Tumour Biol. 2015;36(5):3389-97.

21. Mori S, Usami N, Fukumoto K, Mizuno T, Kuroda H, Sakakura N, et al. The significance of the prognostic nutritional index in patients with completely resected non-small cell lung Cancer. PLoS One. 2015;10(9):e0136897.

22. Feng JF, Chen QX. Significance of the prognostic nutritional index in patients with esophageal squamous cell carcinoma. Ther Clin Risk Manag. 2014; 10:1-7

23. Fan L, Wang X, Chi C, Wang Y, Cai W, Shao X, et al. Prognostic nutritional index predicts initial response to treatment and prognosis in metastatic castration-resistant prostate cancer patients treated with abiraterone. Prostate. 2017;77(12):1233-41.

24. Mottet N, Bellmunt J, Bolla M, et al. EAU-ESTRO-SIOG guidelines on prostate Cancer. Part 1: screening, diagnosis, and local treatment with curative intent. Eur Urol. 2017;71(4):618-29.

25. Cornford P, Bellmunt J, Bolla M, et al. EAU-ESTRO-SIOG guidelines on prostate Cancer. Part II: treatment of relapsing, metastatic, and castrationresistant prostate Cancer. Eur Urol. 2017:71(4):630-42.

26. Wang Y, Yin W, Wang Z, Huang J, Pan J, Zhu Y, Xu F, Shao X, Sha J, Cai Y, Liu $\mathrm{Q}$, Dong $\mathrm{B}, \mathrm{Xue} \mathrm{W}$. And $Y$ Huang. Pretreatment plasma fibrinogen as an independent prognostic indicator of prostate cancer patients treated with androgen deprivation therapy. Prostate Cancer Prostatic Dis. 2016;19(2):209-15.

27. Chen W, Zheng R, Baade PD, Zhang S, Zeng H, Bray F, et al. Cancer statistics in China, 2015. CA Cancer J Clin. 2016;66(2):115-32.

28. Van Cutsem E, Arends J. The causes and consequences of cancer-associated malnutrition. Eur J Oncol Nurs. 2005;9(Suppl 2):S51-63.

29. Caccialanza R, Pedrazzoli P, Cereda E, Gavazzi C, Pinto C, Paccagnella A, et al. Nutritional support in Cancer patients: a position paper from the Italian Society of Medical Oncology (AIOM) and the Italian Society of Artificial Nutrition and Metabolism (SINPE). J Cancer. 2016;7(2):131-5.

30. Gupta D, Lis CG. Pretreatment serum albumin as a predictor of cancer survival: a systematic review of the epidemiological literature. Nutr J. 2010;9:69.

31. Mantovani A, Allavena P, Sica A, Balkwill F. Cancer-related inflammation. Nature. 2008;454(7203):436-44.

32. Nakashima J, Tachibana M, Ueno M, Miyajima A, Baba S, Murai M. Association between tumor necrosis factor in serum and cachexia in patients with prostate cancer. Clin Cancer Res. 1998;4(7):1743-8.

33. Dunn GP, Old LJ, Schreiber RD. The immunobiology of cancer immunosurveillance and immunoediting. Immunity. 2004;21(2):137-48.

34. Huang SH, Waldron JN, Milosevic M, Shen X, Ringash J, Su J, et al. Prognostic value of pretreatment circulating neutrophils, monocytes, and lymphocytes in Oropharyngeal Cancer stratified by human papillomavirus status. Cancer. 2015;121(4):545-55.

35. Roth MD, Harui A. Human tumor infiltrating lymphocytes cooperatively regulate prostate tumor growth in a humanized mouse model. J Immunother Cancer. 2015;3:12.

36. Sun P, Zhang F, Chen C, An X, Li YH, Wang FH, et al. Comparison of the prognostic values of various nutritional parameters in patients with esophageal squamous cell carcinoma from southern China. J Thorac Dis. 2013;5(4):484-91.

37. Kinoshita A, Onoda H, Imai N, Iwaku A, Oishi M, Fushiya N, et al. Comparison of the prognostic value of inflammation-based prognostic scores in patients with hepatocellular carcinoma. Br J Cancer. 2012;107(6):988-93.

38. Wang DS, Luo HY, Qiu MZ, Wang ZQ, Zhang DS, Wang FH, et al. Comparison of the prognostic values of various inflammation based factors in patients with pancreatic cancer. Med Oncol. 2012;29(5):3092-100.

39. Sun $\mathrm{K}, \mathrm{Chen} \mathrm{S}, \mathrm{Xu} J, \mathrm{Li} \mathrm{G}, \mathrm{He} \mathrm{Y}$. The prognostic significance of the prognostic nutritional index in cancer: a systematic review and metaanalysis. J Cancer Res Clin Oncol. 2014;140(9):1537-49.

40. Watanabe M, Iwatsuki M, Iwagami S, Ishimoto T, Baba Y, Baba H. Prognostic nutritional index predicts outcomes of gastrectomy in the elderly. World J Surg. 2012;36(7):1632-9.

\section{Publisher's Note}

Springer Nature remains neutral with regard to jurisdictional claims in published maps and institutional affiliations. 